\title{
Entanglement and the ticking of the clock
}

\author{
A. Valdés-Hernández ${ }^{*}\left[\right.$, C. G. Maglione ${ }^{2}$, A. P. Majtey,2,0, A. R. Plastino ${ }^{4}$ \\ ${ }^{1}$ Universidad Nacional Autónoma de México, Instituto de Física, Apartado Postal 20-364, Ciudad de México, México. \\ ${ }^{2}$ Universidad Nacional de Córdoba, Facultad de Matemática, Astronomía, Física y Computación, Córdoba, Argentina. \\ ${ }^{3}$ Instituto de Física Enrique Gaviola, Consejo Nacional de Investigaciones Científicas y Técnicas de la República Argentina, \\ Córdoba, Argentina. \\ ${ }^{4}$ Universidad Nacional del Noroeste de la Prov. de Buenos Aires, CeBio y Departamento de Ciencias Básicas, Junin, Argentina.
}

Received on November 10, 2019. Revised on February 25, 2020. Accepted on February 28, 2020.

\begin{abstract}
The concept of entanglement is at the heart of quantum physics. It plays a central role in all quantum phenomena involving composite systems. Interestingly, there is an intriguing idea that has attracted considerable attention recently, according to which quantum entanglement may also be essential for understanding the very emergence of time and dynamical evolution. Within this point of view, sometimes referred to as the timeless picture of quantum dynamics, the Universe is regarded as consisting of a clock and a system (or "rest of the Universe") that are jointly in a stationary quantum state, and time evolution arises as an emergent phenomenon rooted at the entanglement between the clock and the system. Here we provide a pedagogical and self-contained exposition, at the upper undergraduate level, of the role of entanglement in this timeless evolution approach to quantum mechanics. In particular, we give a detailed explanation of how the entanglement between the clock and the system is directly and quantitatively related to the average distinguishability between the states of the system at different times.
\end{abstract}

Keywords: quantum dynamics, quantum entanglement, evolution without evolution

\section{Introduction}

Modern physics recognizes quantum entanglement as one of the most intriguing and significant physical phenomena. Once circumscribed to the foundational discussions of quantum mechanics, the applications of entanglement were developed and expanded into numerous fruitful directions, the most spectacular being perhaps those within the realm of quantum information. In a few decades, entanglement evolved from being a puzzling manifestation of the weirdness of quantum mechanics to become a useful resource in promising quantum technologies, and a central phenomenon for the understanding of many aspects of the workings of Nature [1-3]. As a result, entanglement has gained a more prominent role in classrooms, and efforts have been devoted to incorporate the diverse facets of the concept of entanglement to the teaching of quantum mechanics 4 13. However, there is an interesting aspect of quantum theory - known as the timeless picture of quantum mechanics - in which entanglement is crucial for solving the so-called time problem, that has received little or no attention from a pedagogical point of view. With the purpose of enriching the vision of entanglement and the possibilities it offers, it is our aim here to introduce the non-specialized reader to the role played by the entanglement within the timeless approach of quantum mechanics.

*Correspondence email address: andreavh@fisica.unam.mx
The time problem refers to the fact that the introduction of time constitutes a problem at a fundamental level for any theory endowed with dynamical laws. As is well-known, classical mechanics resorts to an extrinsic and privileged parameter $t$, in terms of which all the equations of motion are formulated. On the other hand, and in sharp contrast with this fundamental role played by $t$, from the practical point of view the parameter $t$ is nothing more than the coordinate of a pointer in an appropriate clock (that is, $t$ represents the position of the clock's hands). The tension between these two aspects of time constitutes part of the time problem and, as we shall see, also points towards a possible way of solving it. Quantum mechanics inherits from classical mechanics the time problem, incorporating the extrinsic temporal parameter in, for example, the Schrödinger (time-dependent) equation. This way of introducing time into physical theories has bothered many physicist, mainly because it puts time as an immutable, unexplained variable. The discomfort becomes particularly accentuated when it comes to quantum mechanics, in which the dynamical variables, such as position and momentum, are promoted to Hermitian operators in an appropriate Hilbert space. Time, on the contrary, is not regarded as an observable, and its meaning within quantum theory is a subtle matter 14,17 .

The time problem is central to modern physics: it impacts quantum mechanics, cosmology, and general relativity. In an attempt to tackle it by incorporating time into a completely quantum context, Page and Wooters 
have proposed the timeless approach $\sqrt{14,15}$. It is physically motivated by the observation that the notion of time, when understood as a parameter that indicates the state of the clock hands, can be substituted by the notion of quantum correlations between the clock $(C)$ and the physical system of interest $(R)$. The 'evolution' of $R$ thus simply reflects the fact that for different states of $C$ there correspond different states of $R$, while the whole $U=R+C$ remains in a single static state. In this sense, in the timeless approach both the dynamics and time emerge from non-local correlations, and contrary to Newtonian mechanics, motion is not assumed as a primitive, but secondary, concept [17].

In more formal terms, the timeless proposal considers the whole universe $U$ as a closed composite quantum system comprising two non-interacting subsystems: the clock $C$ and the rest $R$, and assumes that the global system is in a static state (eigenstate of the total Hamiltonian with corresponding null eigenvalue, consistently with the Wheeler-DeWitt equation 17, 18, developed within the quantum theory of gravity). For an observer in $R$, the notion of time (or temporal flux) emerges from this 'frozen' state as a result of the quantum entanglement between $C$ and $R$, and the parameter $t$ is identified with the values of an appropriate observable of the clock (the position of its hands). According to this idea, time (and the concomitant dynamics) would be an emergent property rooted at the entanglement between the dynamical system $(R)$ and a ticking apparatus.

The timeless formalism thus brings together the emergence of time - the parameter characterizing evolution and change - and the notion of entanglement, the most paradigmatic feature of composite quantum systems. Moreover, it places entanglement as the fundamental phenomenon that gives rise to quantum evolution. The timeless picture of quantum mechanics has attracted considerable attention recently $19 \sqrt{23}$, constituting an intriguing line of enquiry within the active field of research devoted to quantum entanglement. This timeless approach, and its connection with entanglement, constitutes also a stimulating subject for a student project. It offers a unique opportunity for students to discuss a cutting-edge topic on fundamental physics. It has scientific, philosophical, and historical relevance, and (as shown in the present work) can be treated in an quantitative, yet comprehensible, way.

In order to make accessible the relation between entanglement and the timeless quantum formalism to a broad non-specialized audience, including an upper-level undergraduate physics student, we provide here an introductory discussion on this matter. Based on one of the most widely used quantitative indicators of entanglement, we first focus on the analysis of the entanglement between the clock and the remaining subsystem. Special attention is then payed to the physical interpretation of the 'amount of entanglement' as a quantitative indicator of the average distinguishability between the states that the system visits at different times. This time-averaged distinguishability is explicitly calculated for a general state of a system with a discrete (though not necessarily finite) energy spectrum.

The paper is organized as follows. In Section II we review some basic features of a simple, yet useful and widely used quantitative indicator of entanglement for pure states of a bipartite quantum system, based on the linear entropy. In Sec. 3 we introduce the main assumptions in the timeless formalism, and make explicit the relation between entanglement and evolution. In Sec. 4 we apply the indicator of entanglement based on the linear entropy to evaluate the entanglement between the clock $C$ and the system $R$, and explore its connection with the time evolution of $R$. Specifically, we show that the entanglement between $C$ and $R$ is closely related to the average distinguishability of the states of $R$ at different times. Section 5 is devoted to calculate explicitly the entanglement for an arbitrary quantum state, and to discuss some relevant examples. Finally, some conclusions are drawn in Sec. 6

\section{Quantitative indicator of entanglement}

According to quantum mechanics, the maximal knowledge that Nature allows us to have about the state of a physical system is given by a pure quantum state. This is mathematically represented by a wave function or, more abstractly, by a normalized vector in an appropriate Hilbert space. One of the most counter-intuitive manifestations of the quantumness of composite quantum systems is that it is possible to have maximal knowledge of a total bipartite system without having maximal knowledge of its parts 8]. When this is the case, we say that the corresponding pure quantum state of the bipartite system is entangled: the composite system is globally described by a pure state, yet the states of either of its subsystems cannot be pure, but instead are described by mixed states. The degree of mixedness of these marginal states constitutes thus an indicator of entanglement of the global, pure state. Hence, it is possible to resort to measures that quantify such degree of mixedness to construct quantitative indicators of the amount of entanglement in a pure (bipartite) state [1]. Now, a convenient measure for the degree of mixedness of the subsystems' marginal states is given by the linear entropy of the associated density matrices (see [11] for a didactic discussion). In what follows we are going to briefly review this quantitative indicator of entanglement for pure states of bipartite quantum systems.

We start by considering a composite quantum system $A+B$ constituted by two subsystems, $A$ and $B$. Let

$$
|\Psi\rangle=\int \Psi\left(\boldsymbol{x}_{1}, \boldsymbol{x}_{2}\right)\left|\boldsymbol{x}_{1}\right\rangle\left|\boldsymbol{x}_{2}\right\rangle d \boldsymbol{x}_{1} d \boldsymbol{x}_{2}
$$


be a pure state of $A+B$. Here $\left\{\left|\boldsymbol{x}_{1}\right\rangle \otimes\left|\boldsymbol{x}_{2}\right\rangle=\left|\boldsymbol{x}_{1}\right\rangle\left|\boldsymbol{x}_{2}\right\rangle=\right.$ $\left.\left|\boldsymbol{x}_{1}, \boldsymbol{x}_{2}\right\rangle\right\}$ denotes a basis of the Hilbert space of the composite system, namely $\mathcal{H}_{A} \otimes \mathcal{H}_{B}$, where $\mathcal{H}_{A}$ and $\mathcal{H}_{B}$ stand for the Hilbert spaces associated to the subsystems $A$ and $B$, respectively, with corresponding basis $\left\{\left|\boldsymbol{x}_{1}\right\rangle\right\}$ and $\left\{\left|\boldsymbol{x}_{2}\right\rangle\right\}$. The members of these basis are characterized by continuous labels $\boldsymbol{x}_{1} \in \mathbb{R}^{N_{1}}$ and $\boldsymbol{x}_{2} \in \mathbb{R}^{N_{2}}$, and satisfy the standard orthogonality condition $\left\langle\boldsymbol{x} \mid \boldsymbol{x}^{\prime}\right\rangle=$ $\delta\left(\boldsymbol{x}-\boldsymbol{x}^{\prime}\right)$. The subsystems $A$ and $B$ can be, for example, two particles moving, respectively, in their corresponding configuration space of dimension $N_{1}$ and $N_{2}$. As usual, the wave function $\Psi\left(\boldsymbol{x}_{1}, \boldsymbol{x}_{2}\right)=\left\langle\boldsymbol{x}_{1}, \boldsymbol{x}_{2} \mid \Psi\right\rangle$ is normalized to one, that is, $\langle\Psi \mid \Psi\rangle=\int\left|\Psi\left(\boldsymbol{x}_{1}, \boldsymbol{x}_{2}\right)\right|^{2} d \boldsymbol{x}_{1} d \boldsymbol{x}_{2}=1$.

Instead of using the ket $|\Psi\rangle$ to describe a pure state of the system $A+B$, one can use the equivalent description provided by the density matrix

$$
\begin{aligned}
\rho & =|\Psi\rangle\langle\Psi| \\
& =\int\left|\boldsymbol{x}_{1}, \boldsymbol{x}_{2}\right\rangle\left\langle\boldsymbol{x}_{1}, \boldsymbol{x}_{2} \mid \Psi\right\rangle\left\langle\Psi \mid \boldsymbol{x}_{1}^{\prime}, \boldsymbol{x}_{2}^{\prime}\right\rangle\left\langle\boldsymbol{x}_{1}^{\prime}, \boldsymbol{x}_{2}^{\prime}\right| \times \\
& \times d \boldsymbol{x}_{1} d \boldsymbol{x}_{2} d \boldsymbol{x}_{1}^{\prime} d \boldsymbol{x}_{2}^{\prime},
\end{aligned}
$$

with matrix elements

$$
\begin{aligned}
\left\langle\boldsymbol{x}_{1}, \boldsymbol{x}_{2}|\rho| \boldsymbol{x}_{1}^{\prime}, \boldsymbol{x}_{2}^{\prime}\right\rangle & =\left\langle\boldsymbol{x}_{1}, \boldsymbol{x}_{2} \mid \Psi\right\rangle\left\langle\Psi \mid \boldsymbol{x}_{1}^{\prime}, \boldsymbol{x}_{2}^{\prime}\right\rangle \\
& =\Psi\left(\boldsymbol{x}_{1}, \boldsymbol{x}_{2}\right) \Psi^{*}\left(\boldsymbol{x}_{1}^{\prime}, \boldsymbol{x}_{2}^{\prime}\right) .
\end{aligned}
$$

The marginal density matrices $\rho_{A}$ and $\rho_{B}$ describing, respectively, the individual states of the subsystems $A$ and $B$, are

$$
\rho_{A(B)}=\operatorname{Tr}_{B(A)} \rho=\operatorname{Tr}_{B(A)}(|\Psi\rangle\langle\Psi|),
$$

where $\operatorname{Tr}_{i}$ denotes the partial trace over the degrees of freedom of subsystem $i$. The matrix elements of the marginal density matrices are

$$
\begin{aligned}
\left\langle\boldsymbol{x}_{1}\left|\rho_{A}\right| \boldsymbol{x}_{1}^{\prime}\right\rangle & =\int\left\langle\boldsymbol{x}_{1}, \boldsymbol{x}_{2}|\rho| \boldsymbol{x}_{1}^{\prime}, \boldsymbol{x}_{2}\right\rangle d \boldsymbol{x}_{2} \\
& =\int \Psi\left(\boldsymbol{x}_{1}, \boldsymbol{x}_{2}\right) \Psi^{*}\left(\boldsymbol{x}_{1}^{\prime}, \boldsymbol{x}_{2}\right) d \boldsymbol{x}_{2}, \\
\left\langle\boldsymbol{x}_{2}\left|\rho_{B}\right| \boldsymbol{x}_{2}^{\prime}\right\rangle & =\int\left\langle\boldsymbol{x}_{1}, \boldsymbol{x}_{2}|\rho| \boldsymbol{x}_{1}, \boldsymbol{x}_{2}^{\prime}\right\rangle d \boldsymbol{x}_{1} \\
& =\int \Psi\left(\boldsymbol{x}_{1}, \boldsymbol{x}_{2}\right) \Psi^{*}\left(\boldsymbol{x}_{1}, \boldsymbol{x}_{2}^{\prime}\right) d \boldsymbol{x}_{1} .
\end{aligned}
$$

A quantitative indicator $\mathcal{E}[|\Psi\rangle]$ of the amount of entanglement between $A$ and $B$ when the composite system is in the pure state $|\Psi\rangle$, is given by the degree of mixedness exhibited by the marginal density matrices $\rho_{A(B)}$. Such degree of mixedness can be quantified by the linear entropy $S_{L}$ of either one of the two reduced matrices, given by

$$
S_{L}\left[\rho_{A(B)}\right]=1-\operatorname{Tr} \rho_{A(B)}^{2} .
$$

Therefore, we can define the following indicator of entanglement,

$$
\mathcal{E}[|\Psi\rangle]=1-\operatorname{Tr} \rho_{A}^{2}=1-\operatorname{Tr} \rho_{B}^{2}
$$

To obtain an explicit expression for $\mathcal{E}[|\Psi\rangle]$ in terms of the wave function $\Psi\left(\boldsymbol{x}_{1}, \boldsymbol{x}_{2}\right)$ we compute the trace:

$$
\begin{aligned}
\operatorname{Tr} \rho_{A}^{2} & =\int\left\langle\boldsymbol{x}_{1}\left|\rho_{A}^{2}\right| \boldsymbol{x}_{1}\right\rangle d \boldsymbol{x}_{1} \\
& =\int\left\langle\boldsymbol{x}_{1}\left|\rho_{A}\right| \boldsymbol{x}_{1}^{\prime}\right\rangle\left\langle\boldsymbol{x}_{1}^{\prime}\left|\rho_{A}\right| \boldsymbol{x}_{1}\right\rangle d \boldsymbol{x}_{1} d \boldsymbol{x}_{1}^{\prime} \\
& =\int\left|\left\langle\boldsymbol{x}_{1}\left|\rho_{A}\right| \boldsymbol{x}_{1}^{\prime}\right\rangle\right|^{2} d \boldsymbol{x}_{1} d \boldsymbol{x}_{1}^{\prime} .
\end{aligned}
$$

Finally, combining Eqs. (5) and (8), we obtain

$$
\begin{aligned}
\mathcal{E}[|\Psi\rangle] & =1-\operatorname{Tr} \rho_{A}^{2} \\
& =1-\int \Psi\left(\boldsymbol{x}_{1}, \boldsymbol{x}_{2}\right) \Psi^{*}\left(\boldsymbol{x}_{1}^{\prime}, \boldsymbol{x}_{2}\right) \times \\
& \times \Psi^{*}\left(\boldsymbol{x}_{1}, \boldsymbol{x}_{2}^{\prime}\right) \Psi\left(\boldsymbol{x}_{1}^{\prime}, \boldsymbol{x}_{2}^{\prime}\right) d \boldsymbol{x}_{1} d \boldsymbol{x}_{1}^{\prime} d \boldsymbol{x}_{2} d \boldsymbol{x}_{2}^{\prime},
\end{aligned}
$$

which constitutes the desired expression for the entanglement measure $\mathcal{E}[|\Psi\rangle]$ in terms of the wave function $\Psi\left(\boldsymbol{x}_{1}, \boldsymbol{x}_{2}\right)$.

If the wave function $\Psi\left(\boldsymbol{x}_{1}, \boldsymbol{x}_{2}\right)$ is normalized to 1 (as we are assuming) one has $\operatorname{Tr} \rho_{A(B)}^{2} \leq 1$, with the equality satisfied if and only if the matrices $\rho_{A(B)}$ correspond to pure states and, consequently, the global state $|\Psi\rangle$ is separable (that is, non-entangled). It thus follows that the entanglement measure $\mathcal{E}$ satisfies the inequality

$$
0 \leq \mathcal{E}[|\Psi\rangle] \leq 1
$$

and vanishes if and only if the state $|\Psi\rangle$ is non entangled. It should be noticed that though in the above lines we have considered systems with continuous variables, and expressed $\mathcal{E}[|\Psi\rangle]$ in terms of the wave function $\Psi\left(\boldsymbol{x}_{1}, \boldsymbol{x}_{2}\right)$, the entanglement measure (7) is also well-defined - and satisfies the inequality 10 - for pure states of bipartite quantum systems in which $\mathcal{H}_{A}$ and $\mathcal{H}_{B}$ have finite dimensions, as is the case for systems of qubits.

The quantity $\mathcal{E}[|\Psi\rangle]$ is an entanglement monotone (i.e., it does not increase, on average, under local transformations [1]), it vanishes for separable pure states and it admits its largest possible value for maximally entangled pure states. Thus, it complies with basic requirements that any entanglement measure should satisfy [1]. We stress here, however, that the linear entropy provides just one way of quantifying the bipartite entanglement in a pure state. Other quantities exist that serve for that same purpose; a more fundamental one is the von Neumann entropy, defined as

$$
S_{v N}\left[\rho_{A(B)}\right]=-\operatorname{Tr}\left[\rho_{A(B)} \ln \rho_{A(B)}\right] .
$$

Expressing the reduced state, say $\rho_{A}$, in its diagonal form $\rho_{A}=\operatorname{diag}\left(\left\{\lambda_{\alpha}\right\}\right)$, with $\left\{\lambda_{\alpha}\right\}$ the (real) eigenvalues of $\rho_{A}$ satisfying $\sum_{\alpha} \lambda_{\alpha}=1$, the von Neumann entropy expresses as

$$
S_{v N}\left[\rho_{A}\right]=-\sum_{\alpha} \lambda_{\alpha} \ln \lambda_{\alpha}
$$

From Eq. 12 it follows that the von Neumann entropy vanishes if and only if the reduced states $\rho_{A(B)}$ are pure 
(one $\lambda_{\alpha}$ equals 1 and the remaining ones equal 0 ), meaning that $A$ and $B$ are disentangled. Importantly, the von Neumann entropy lies at the basis of the so-called entanglement of formation, one of the most exploited measures of bipartite entanglement in general (mixed) bipartite states. However, in spite of the significance of $S_{v N}$ in certifying and quantifying entanglement, the indicator $\mathcal{E}[|\Psi\rangle]$, based on the linear entropy, has the important computational advantage (both from the analytical and the numerical points of view) that in order to compute it, one does not need to determine the eigenvalues of the marginal density matrix $\rho_{A}$. Moreover, it has been extensively used as a legitimate measure of entanglement 24 29], playing in particular a prominent role in the expression for the so-called concurrence, a widely employed measure of bipartite entanglement (see, for instance, $24,27,28]$ ). Thus, for our present purposes, the simpler linear entropy-based quantitative entanglement indicator suffices. A nice and interesting introduction to entanglement in composite quantum systems with continuous variables, that considers the measure $(9)$, can be found in [4]. The entanglement indicator based on the linear entropy is a particular instance of a more general family of entanglement indicators 29] based on the quantum $S_{q}$ entropies 30 .

Before ending this Section, it is worthwhile stressing that in more general terms, for pure states of the composite system $A+B$, any measure of the degree of mixedness of either of the marginal (reduced) states $\rho_{A}$ or $\rho_{B}$, provides a quantitative indicator of the amount of entanglement between $A$ and $B$. Such measures of mixedness, or generalized entropies, can be expressed in the form $\operatorname{Tr}\left[f\left(\rho_{A(B)}\right)\right]$, where $f(x)$ is a concave function (that is $f^{\prime \prime}(x) \leq 0$ ), such that $f(0)=f(1)=0$. Clearly both the von Neumann entropy and the linear entropy have this form. In the first case we have $f(x)=-x \ln x$, whereas in the second case we have $f(x)=x(1-x)$.

\section{Entanglement and the emergence of evolution}

As a starting point, we consider the Universe as a bipartite quantum system conformed by a clock $(C)$ and the rest $(R)$. The corresponding Hilbert spaces are $\mathcal{H}_{R}$ for the rest of the Universe, $\mathcal{H}_{C}$ for the clock, and the tensor product $\mathcal{H}_{R} \otimes \mathcal{H}_{C}$ for the total system $(U=R+C)$. We shall express a pure state of $U$ in terms of a product orthonormal basis $\{|\boldsymbol{x}\rangle \otimes|t\rangle=|\boldsymbol{x}\rangle|t\rangle\}$, where $\{|\boldsymbol{x}\rangle\}$ and $\{|t\rangle\}$ are orthonormal basis of $\mathcal{H}_{R}$ and $\mathcal{H}_{C}$, respectively. The continuous label $t \in \mathbb{R}$, characterizing the clock's basis states, corresponds to the position of the hands of a clock associated to an observable $\hat{\mathcal{T}}$, that is, $\hat{\mathcal{T}}|t\rangle=t|t\rangle$. On the other hand, the label $\boldsymbol{x}$, characterizing the basis states of $\mathcal{H}_{R}$, represents the position, or any other degree of freedom of the one, or many particles, that conform $R$. In other words, $\boldsymbol{x}$ can be regarded as describing a point in the configuration space associated with the system
$R$ (throughout the paper we will assume that $\boldsymbol{x}$ is a continuous variable, yet it may also denote a discrete one if integrals are properly substituted by discrete sums).

In line with the timeless formalism, we will assume that $U$ is in a pure state $|\Psi\rangle$ such that $\hat{H}_{U}|\Psi\rangle=0$, where $\hat{H}_{U}=\hat{H}_{R} \otimes \mathbb{I}_{C}+\mathbb{I}_{R} \otimes \hat{H}_{C}$ is the total Hamiltonian, with $\hat{H}_{R}$ an arbitrary Hamiltonian of $R$, and $\hat{H}_{C}$ the Hamiltonian of the clock. Furthermore, we will consider that $\hat{H}_{C}$ and the clock's observable $\hat{\mathcal{T}}$ satisfy the commutation relation

$$
\left[\hat{\mathcal{T}}, \hat{H}_{C}\right]=i \hbar .
$$

This amounts to say that $\hat{H}_{C}$ is the generator of displacements in the position of the clock's hands; that is, for an infinitesimal displacement $d t$ it holds that $e^{-i \hat{H}_{C} d t / \hbar}|t\rangle=$ $|t+d t\rangle$, or equivalently, that $i \hbar d|t\rangle / d t=\hat{H}_{C}|t\rangle$. Now, the state of $R$ when the clock reads $t$, that is, its state given that the clock is in $|t\rangle$, is obtained by projecting $|\Psi\rangle$ onto $|t\rangle$, and will be denoted as $\left|\Phi_{t}\right\rangle=\langle t \mid \Psi\rangle$. Under these conditions, we get

$$
\begin{aligned}
\frac{\partial}{\partial t}\left|\Phi_{t}\right\rangle & =\frac{\partial}{\partial t}\langle t \mid \Psi\rangle \\
& =\left(\frac{\partial}{\partial t}\langle t|\right)|\Psi\rangle+\langle t|\left(\frac{\partial}{\partial t}|\Psi\rangle\right) \\
& =\left(\frac{d}{d t}\langle t|\right)|\Psi\rangle \\
& =\frac{i}{\hbar}\left\langle t\left|\hat{H}_{C}\right| \Psi\right\rangle .
\end{aligned}
$$

Writing $\mathbb{I}_{R} \otimes \hat{H}_{C}=\hat{H}_{U}-\hat{H}_{R} \otimes \mathbb{I}_{C}$ and using that $\hat{H}_{U}|\Psi\rangle=0$, Eq. 14 becomes

$$
\begin{aligned}
\frac{\partial}{\partial t}\left|\Phi_{t}\right\rangle & =\frac{i}{\hbar}\left\langle t\left|\left(\hat{H}_{U}-\hat{H}_{R} \otimes \mathbb{I}_{C}\right)\right| \Psi\right\rangle \\
& =\frac{-i}{\hbar}\left\langle t\left|\hat{H}_{R} \otimes \mathbb{I}_{C}\right| \Psi\right\rangle \\
& =\frac{-i}{\hbar} \hat{H}_{R}\langle t \mid \Psi\rangle \\
& =\frac{-i}{\hbar} \hat{H}_{R}\left|\Phi_{t}\right\rangle
\end{aligned}
$$

whence $\left|\Phi_{t}\right\rangle$ obeys

$$
i \hbar \frac{\partial}{\partial t}\left|\Phi_{t}\right\rangle=\hat{H}_{R}\left|\Phi_{t}\right\rangle
$$

That is, the relative state of the rest of the Universe is found to satisfy the (time-dependent) Schrödinger equation. In other words, the usual dynamical scenario - embodied in the time-dependent Schrödinger equationensues from the static image of the non-evolving state $|\Psi\rangle$.

A few comments regarding the eigenvalue spectra of the operators $\hat{\mathcal{T}}$ and $\hat{H}_{C}$ is in place here. Both these observables have continuous spectra; however, since the complete system is assumed to be in a zero-energy eigenstate of the total Hamiltonian, if $\hat{H}_{R}$ has discrete eigenvalues $\left\{E_{n}\right\}$ with $n=0,1,2, \ldots$ (as will be assumed below), 
then the allowed eigenvalues of $\hat{H}_{C}$ also form a discrete set, namely $\left\{-E_{n}\right\}$, and the corresponding eigenstates are thus $\left\{e^{-i E_{n} t / \hbar}\right\}$. This means that we are working with an effectively discrete Hilbert space for the clock, spanned by this discrete set of states. Notice that this is similar to what occurs when considering the position and the momentum of a particle in a one-dimensional box. The operators $\hat{x}$ and $\hat{p}$ also satisfy a relation of the form (13) (they are canonical conjugate variables), and by imposing the appropriate boundary conditions on the (impenetrable) walls, the accesible eigenvalues of the momentum form a discrete set, $\left\{p_{n}\right\}$, and the eigenfunctions that span the Hilbert space of the system are given by $\left\{e^{-i p_{n} x / \hbar}\right\}$. Of course, the similarity is only formal, since in the present case the (effective) discrete spectra of $\hat{H}_{C}$ is not determined by any boundary condition, but rather by the condition of total zero energy of the $R+C$ composite system.

In what follows we shall consider the behaviour of the system when the hands of the clock acquire values in the interval $[0, T]$, and write $|\Psi\rangle$ (the global state of $U$ ) as

$$
|\Psi\rangle=\frac{1}{\sqrt{T}} \int \Psi(\boldsymbol{x}, t)|\boldsymbol{x}\rangle|t\rangle d \boldsymbol{x} d t .
$$

This state is properly normalized, both spatially and temporally, that is

$$
\langle\Psi \mid \Psi\rangle=\frac{1}{T} \int_{0}^{T} \underbrace{\left(\int|\Psi(\boldsymbol{x}, t)|^{2} d \boldsymbol{x}\right)}_{=1} d t=1 .
$$

The relative state $\left|\Phi_{t}\right\rangle$ thus reads

$$
\left|\Phi_{t}\right\rangle=\langle t \mid \Psi\rangle=\frac{1}{\sqrt{T}} \int \Psi(\boldsymbol{x}, t)|\boldsymbol{x}\rangle d \boldsymbol{x}=\frac{1}{\sqrt{T}}\left|\widetilde{\Phi}_{t}\right\rangle,
$$

where we defined

$$
\left|\widetilde{\Phi}_{t}\right\rangle=\sqrt{T}\left|\Phi_{t}\right\rangle=\int \Psi(\boldsymbol{x}, t)|\boldsymbol{x}\rangle d \boldsymbol{x}
$$

Therefore, $\frac{1}{\sqrt{T}} \Psi(\boldsymbol{x}, t)=\left\langle\boldsymbol{x} \mid \Phi_{t}\right\rangle$ is the wave function corresponding to the state $\left|\Phi_{t}\right\rangle$, whose norm is given by

$$
\left\langle\Phi_{t} \mid \Phi_{t}\right\rangle=\frac{1}{T} \int|\Psi(\boldsymbol{x}, t)|^{2} d \boldsymbol{x}=\frac{1}{T},
$$

whence $\left|\widetilde{\Phi}_{t}\right\rangle$ stands for the the normalized relative state, satisfying

$$
\left\langle\widetilde{\Phi}_{t} \mid \widetilde{\Phi}_{t}\right\rangle=T\left\langle\Phi_{t} \mid \Phi_{t}\right\rangle=1
$$

Considering values of $t$ within a finite interval $[0, T]$ corresponds to studying a part of the history of the Universe that, from the standard time-based perspective, is perceived as having a finite duration $T$. To have a finite range of values $[0, T]$ for the clock's position observable, and to have the wave function normalized within that finite interval, can be regarded as the result of having measured the observable (proyector) $\Pi=\int_{0}^{T}|t\rangle\langle t| d t$, post-selecting the measurement result 1 . In other words, states normalized within the finite range $[0, T]$ are the result of proyecting the state of the Universe onto the subspace spanned by eigenstates of $\hat{\mathcal{T}}$ with eigenvalues $t \in[0, T]$.

In terms of the wave function $\Psi(\boldsymbol{x}, t)$, Eq. 16 reads

$$
i \hbar \frac{\partial}{\partial t} \Psi(\boldsymbol{x}, t)=\hat{H}_{R} \Psi(\boldsymbol{x}, t)
$$

hence, if condition 21 holds for an initial time $t=0$, it will hold for all $t$. Now, from Eq. (23) it follows that if $R$ and $C$ are non-entangled, so that $\Psi(\boldsymbol{x}, t)$ is a factorizable state of the form $\Psi(\boldsymbol{x}, t)=\Psi_{R}(\boldsymbol{x}) \Psi_{C}(t)$, then $\Psi_{R}(\boldsymbol{x})$ is an eigenstate of $\hat{H}_{R}$, consequently $\Psi(\boldsymbol{x}, t)$ is a stationary state, and $R$ does not evolve. Thus, the essential point is that for $\Psi(\boldsymbol{x}, t)$ to evolve, the rest of the universe and the clock must be entangled, that is, $\Psi(\boldsymbol{x}, t)$ must be a non-factorizable function of the variables $\boldsymbol{x}$ and $t$, which in turn requires that the zero eigenvalue of $\hat{H}_{U}$ is degenerate (the higher the degeneracy, the richer the evolution will be). To investigate such intimate relation between entanglement and time evolution is the aim of the following sections.

Now that the main ingredients of the timeless picture of quantum mechanics have been introduced, we can reconsider the timeless approach from an intuitive, qualitative point of view. As we have already seen, in the timeless approach the system $R$ under consideration and the clock $C$ are jointly in a stationary quantum pure state described by the wave function $\Psi(\boldsymbol{x}, t)$. This wave function describes in a static way all that happens to $C$ and $R$ at all times $t$ and at every location $\boldsymbol{x}$. That is, $\Psi(\boldsymbol{x}, t)$ is a static mathematical object codifying all that happens "everywhere and everywhen". In this sense, the timeless picture of quantum mechanics can be regarded as a quantum-mechanical version of the "block universe" picture of time in classical physics 31. According to this latter picture, all that has happened in the past and all that will happen in the future exists in a "frozen" block universe that, together with the multi-dimensional coordinates $\boldsymbol{x}$ describing the configuration space of the system $R$, incorporates a new dimension associated with the time parameter $t$.

Now, in the quantum timeless picture, $t$ is not regarded as a parameter, but as the coordinate describing the clock system $C$. As we have already mentioned, if the system $R$ is perceived as evolving (in the usual sense), then the joint pure state describing the composite $(R+C)$ system has to be entangled. This means that, if $R$ evolves, then, from the timeless point of view, neither of the subsystems $R$ and $C$ can be described by a pure state. Watching the quantum block universe "from the outside", so to speak, one cannot associate a pure state to either $R$ or $C$. In other words, even having a maximum knowledge about the state of the total $R+C$ system, one cannot have complete knowledge about either one of the parts [8]. To describe the subsystems $R$ and $C$ we need the 
corresponding density matrices $\rho_{R}$ and $\rho_{C}$. The marginal density matrix $\rho_{R}$ has a clear intuitive meaning. We shall consider that the global wave function $\Psi(\boldsymbol{x}, t)$ is defined over a finite time interval $[0, T]$. The matrix $\rho_{R}$ results from tracing the global state $\rho=|\Psi\rangle\langle\Psi|$ over the degrees of freedom of the clock $C$, that is, over the coordinate $t \in[0, T]:$

$$
\begin{aligned}
\rho_{R}=\operatorname{Tr}_{C} \rho & =\int_{0}^{T}\langle t \mid \Psi\rangle\langle\Psi \mid t\rangle d t \\
& =\int_{0}^{T}\left|\Phi_{t}\right\rangle\left\langle\Phi_{t}\right| d t \\
& =\frac{1}{T} \int_{0}^{T}\left|\widetilde{\Phi}_{t}\right\rangle\left\langle\widetilde{\Phi}_{t}\right| d t
\end{aligned}
$$

Notice that Eq. 21 guarantees the normalization condition $\operatorname{Tr} \rho_{R}=1$, required by any density matrix describing a physical state.

Equation (24) shows that the marginal density matrix $\rho_{R}$ is given by a statistical mixture of all the (normalized, pure) states $\left|\widetilde{\Phi}_{t}\right\rangle$ that describe the system $R$ at different times within the interval $[0, T]$. That is, $\rho_{R}$ is equal to the time average of the evolving state $\left|\widetilde{\Phi}_{t}\right\rangle$ over the interval $[0, T]$. This state of affairs is essentially quantum-mechanical and does not have a counterpart within the classical block universe picture. This suggests that quantum mechanics is perhaps better adapted to the block universe picture than classical mechanics. Now, the mixed state described by $\rho_{R}$ has a degree of mixture that can be assessed quantitatively by various appropriate measures. These measures of mixedness also provide indicators of the degree of entanglement that exists between the system $R$ and the clock $C$.

The measures of the degree of mixedness of $\rho_{R}$ are related to the effective number of distinguishable pure states that contribute to $\rho_{R}$. For instance, the participation ratio of $\rho_{R}$, given by $1 / \operatorname{Tr}\left(\rho_{R}^{2}\right)$, constitutes a direct measure of the effective number of distinguishable states in $\rho_{R}$. On the other hand, the von Neumann entropy $S_{v N}\left[\rho_{R}\right]$ can be interpreted as an effective indicator of the (logarithm of the) number of distinguishable states entering in the decomposition of a statistical mixture described by $\rho_{R}$. Other entropic measures evaluated on $\rho_{R}$ admit similar interpretations. It is thus intuitively clear that, since $\rho_{R}$ coincides with the time average of the evolving state $\left|\widetilde{\Phi}_{t}\right\rangle$, the measures of the degree of mixedness of $\rho_{R}$ give us a quantitative estimate of the degree of diversity of (or amount of distinguishability between) the different states that the system $R$ visits during the interval $[0, T]$.

In summary, the degree of mixedness of $\rho_{R}$, as measured by a set of different indicators — including the linear or the von Neumann entropy - gives us an estimate of two different things: the amount of entanglement between $C$ and $R$, and the distinguishability of the states of $R$ at different times, during the interval $[0, T]$. Thus, we see that there is a close connection between the evolution of $R$ and the entanglement between $C$ and $R$ : both these phenomena have as a consequence that the quantum state $\rho_{R}$ of the system $R$ is mixed. The more $\rho_{R}$ is mixed, the greater the diversity of states that $R$ visits during its evolution, and the more $R$ is entangled with C.

\section{Quantifying the entanglement between the clock $C$ and the rest of the Universe $R$}

With the aim of exhibiting the tight relation between entanglement and evolution, we shall consider, as before, a finite time interval $[0, T]$ during which the evolution takes place. Moreover, for analyzing the entanglement between $C$ and $R$ we adopt the entanglement indicator discussed in Section II. Therefore, in what follows we will focus on the quantity

$$
\mathcal{E}=1-\operatorname{Tr} \rho_{R}^{2},
$$

with $\rho_{R}$ the marginal density matrix of $R$. The matrix elements of $\rho_{R}$ are given by

$$
\left\langle\boldsymbol{x}\left|\rho_{R}\right| \boldsymbol{x}^{\prime}\right\rangle=\frac{1}{T} \int_{0}^{T} \Psi(\boldsymbol{x}, t) \Psi^{*}\left(\boldsymbol{x}^{\prime}, t\right) d t .
$$

This is an instance of the general expression (5), with $A$ and $B$ substituted by $R$ and $C$, the basis $\left|\boldsymbol{x}_{2}\right\rangle$ substituted by the basis $|t\rangle$ of the clock, and $\Psi$ substituted by the global function $\frac{1}{\sqrt{T}} \Psi(\boldsymbol{x}, t)$, properly normalized in space and time during the chosen time interval.

Recall that, according to Eq. 24, the marginal density matrix $\rho_{R}$ can be interpreted as the time average, over the interval $[0, T]$, of the normalized state $\left|\widetilde{\Phi}_{t}\right\rangle\left\langle\widetilde{\Phi}_{t}\right|$. Further, $\rho_{R}$ is a statistical superposition of the states $\left|\widetilde{\Phi}_{t}\right\rangle\left\langle\widetilde{\Phi}_{t}\right|$ corresponding to different values of $t$ (as opposed to a coherent quantum superposition). It is also worth to emphasize that the density matrices $\rho_{R}$ and $\left|\widetilde{\Phi}_{t}\right\rangle\left\langle\widetilde{\Phi}_{t}\right|$, though both referring to system $R$, represent different states. The latter is obtained via a projective operation. Up to a constant factor, it represents the state of $R$ conditioned to the state $|t\rangle$ of the clock, and is a pure state that evolves unitarily as $t$ flows. In its turn, $\rho_{R}$ is obtained via a partial trace. It represents a time-averaged state (over the interval $[0, T]$ ) and, in general, is a mixed state that does not evolve unitarily with the ticking of the clock.

The entanglement measure 25 is therefore given by

$$
\begin{aligned}
\mathcal{E}= & 1-\operatorname{Tr} \rho_{R}^{2} \\
= & 1-\frac{1}{T^{2}} \int_{0}^{T} d t \int_{0}^{T} d t^{\prime} \int \Psi(\boldsymbol{x}, t) \Psi^{*}\left(\boldsymbol{x}^{\prime}, t\right) \times \\
& \times \Psi^{*}\left(\boldsymbol{x}, t^{\prime}\right) \Psi\left(\boldsymbol{x}^{\prime}, t^{\prime}\right) d \boldsymbol{x} d \boldsymbol{x}^{\prime}
\end{aligned}
$$

which constitutes a particular example of Eq. (9). Expression 27) can be expressed in a more compact way as

$$
\mathcal{E}(T)=1-\frac{1}{T^{2}} \int_{0}^{T} \int_{0}^{T}\left|\left\langle\widetilde{\Phi}_{t} \mid \widetilde{\Phi}_{t^{\prime}}\right\rangle\right|^{2} d t d t^{\prime}
$$


It is worthwhile stressing that the dependence of the entanglement $\mathcal{E}$ on a duration of time $T$ (instead of on time $t$ ) originates in Eq. (24), where the complete density matrix $|\Psi\rangle\langle\Psi|$ is (partially) traced over the degrees of freedom of the clock, that acquire values precisely in the interval $[0, T]$. In fact, this partial trace operation over the degree of freedom $t$ implies that the reduced density matrix $\rho_{R}$, hence $\mathcal{E}$, will not be a function of the variable $t$. Recalling that, as stated below Eq. (26), the marginal density matrix $\rho_{R}$ can be interpreted as the average state over the period $[0, T]$, the quantity $\mathcal{E}(T)$ can be interpreted as the average entanglement in such period.

The second term on the right-hand side of Eq. (28) is a time average, over the interval $[0, T]$, of the overlap $\left|\left\langle\widetilde{\Phi}_{t} \mid \widetilde{\Phi}_{t^{\prime}}\right\rangle\right|^{2}$ between the states $|\widetilde{\Phi}\rangle$ at different instants. Now, the overlap indicates how indistinguishable the quantum state $\left|\widetilde{\Phi}_{t}\right\rangle$ is from the state $\left|\widetilde{\Phi}_{t^{\prime}}\right\rangle$ (zero overlap corresponds to perfectly distinguishable states, whereas overlap equal to one corresponds to identical —up to a global phase - states). Consequently, it is intuitively clear that $\mathcal{E}$ can be interpreted as a quantitative measure of how varied is the life of the state $\left|\widetilde{\Phi}_{t}\right\rangle$ (or equivalently, the unnormalized state $\left.\left|\Phi_{t}\right\rangle\right)$ over the interval $[0, T]$. Indeed, a varied life is such that each moment is different (that is, highly distinguishable) from any other moment. That corresponds to high (close to 1 ) values of $\mathcal{E}$. On the contrary, a monotonous life is one where each moment is similar to (little distinguishable from) any other moment, leading to a low value of $\mathcal{E}$.

The quantity (28) therefore provides a direct visualization of the intimate connection between time evolution and entanglement: on one hand, it is a quantitative indicator of the entanglement between the rest of the Universe and the clock, during the time interval $[0, T]$. On the other hand, it constitutes a quantitative measure of the degree of variety exhibited by the evolving state $\left|\Phi_{t}\right\rangle$ during that same period. The interpretation of the righthand side of $(28)$ as a measure of the degree of variety of the system's state over the interval $[0, T]$, is justified by its relation with the mean overlap (or fidelity) $\left|\left\langle\widetilde{\Phi}_{t} \mid \widetilde{\Phi}_{t^{\prime}}\right\rangle\right|^{2}$, as explained in the last paragraph, implying that for high values of 28 the state $\left|\widetilde{\Phi}_{t}\right\rangle$ is, on average, highly distinguishable from any other state $\left|\widetilde{\Phi}_{t^{\prime}}\right\rangle$, whereas low values of 28 indicate that $\left|\widetilde{\Phi}_{t}\right\rangle$, on average, does not differ much from $\left|\widetilde{\Phi}_{t^{\prime}}\right\rangle$.

\section{Explicit form of the entanglement between $C$ and $R$}

To further analyze $\mathcal{E}$ it is necessary to actually evaluate this measure as a function of $T$ in the general case, in which the wave function $\Psi(\boldsymbol{x}, t)$ evolves according to Eq. (23). For this purpose we start form the expression 20] to write

$$
\left\langle\widetilde{\Phi}_{t} \mid \widetilde{\Phi}_{t^{\prime}}\right\rangle=\int \Psi^{*}(\boldsymbol{x}, t) \Psi\left(\boldsymbol{x}, t^{\prime}\right) d \boldsymbol{x}
$$

and expand $\Psi(\boldsymbol{x}, t)$ in terms of the (orthonormal) eigenstates of $\hat{H}_{R}, \hat{H}_{R} \varphi_{n}(\boldsymbol{x})=E_{n} \varphi_{n}(\boldsymbol{x})$, as follows:

$$
\Psi(\boldsymbol{x}, t)=\sum_{n} a_{n} e^{-i E_{n} t / \hbar} \varphi_{n}(\boldsymbol{x}),
$$

with $\sum_{n}\left|a_{n}\right|^{2}=1$.

The overlap 29 thus rewrites as

$$
\begin{aligned}
\left\langle\widetilde{\Phi}_{t} \mid \widetilde{\Phi}_{t^{\prime}}\right\rangle & =\sum_{n m} a_{n}^{*} a_{m} e^{i\left(E_{n} t-E_{m} t^{\prime}\right) / \hbar} \int \varphi_{n}^{*}(\boldsymbol{x}) \varphi_{m}(\boldsymbol{x}) d \boldsymbol{x} \\
& =\sum_{n m} a_{n}^{*} a_{m} e^{\frac{i}{\hbar}\left(E_{n} t-E_{m} t^{\prime}\right)} \delta_{n m} \\
& =\sum_{n}\left|a_{n}\right|^{2} e^{\frac{i}{\hbar} E_{n}\left(t-t^{\prime}\right)},
\end{aligned}
$$

whence Eq. 28) gives

$$
\begin{aligned}
\mathcal{E}(T) & =1-\frac{1}{T^{2}} \sum_{n m}\left|a_{n} a_{m}\right|^{2} \int_{0}^{T} \int_{0}^{T} d t d t^{\prime} e^{\frac{i}{\hbar}\left(E_{n}-E_{m}\right)\left(t-t^{\prime}\right)} \\
& =1-2 \sum_{n m}\left|a_{n} a_{m}\right|^{2} \frac{\left[1-\cos \left(\omega_{n m} T\right)\right]}{\left(\omega_{n m} T\right)^{2}} \\
& =1-\sum_{n m}\left|a_{n} a_{m}\right|^{2} \operatorname{sinc}^{2}\left(\omega_{n m} T / 2\right)
\end{aligned}
$$

where we defined $\omega_{n m}=\left|E_{n}-E_{m}\right| / \hbar$.

Using that $\sum_{n m}\left|a_{n} a_{m}\right|^{2}=1$, and separating the sum into those terms for which $E_{n}=E_{m}$ (or $\omega_{n m}=0$ ), and those that satisfy $E_{n} \neq E_{m}\left(\right.$ or $\left.\omega_{n m} \neq 0\right)$, we are led to the following expression

$$
\mathcal{E}(T)=\sum_{\substack{n m \\\left(\omega_{n m} \neq 0\right)}}\left|a_{n} a_{m}\right|^{2}\left[1-\operatorname{sinc}^{2}\left(\omega_{n m} T / 2\right)\right],
$$

which gives $\mathcal{E}(T)$ once the initial state $\Psi(\boldsymbol{x}, 0)$ (which determine the expansion coefficients $a_{n}$ ) is determined. Equation (33) shows that the entanglement is not a monotonous function of $T$, so the life of $\left|\Phi_{t}\right\rangle$ in the interval $[0, T]$ exhibits ups and downs which are determined not only by $T$, but also by the transition frequencies, or rather the accesible states that $R$ can visit during its evolution.

\subsection{Maximal and minimal entanglement}

According to Eq. 33 , the maximum value of $\mathcal{E}$ is

$$
\mathcal{E}_{\max }=\sum_{\substack{n m \\\left(\omega_{n m} \neq 0\right)}}\left|a_{n} a_{m}\right|^{2}=1-\sum_{\substack{n m \\\left(\omega_{n m}=0\right)}}\left|a_{n} a_{m}\right|^{2}
$$

and is attained at the zeros of sinc $\left(\omega_{n m} T / 2\right)$, which occur in the limit $T \rightarrow \infty$, or whenever

$$
T=2 \pi l_{n m} / \omega_{n m}
$$


with $l_{n m}=1,2, \ldots$ Since $\omega_{n m}$ varies in general from term to term in the sum in 33 whereas $T$ is a fixed quantity, the condition (35) can be automatically met (assuming no degeneracies) when: i) There is a single transition frequency $\omega_{n m}$, that is, when the expansion 30 involves two terms only, so that the system is a two-level system. In this case $l_{n m}$ can be any positive integer, and $T$ is a multiple of the transition period. ii) The system is an harmonic oscillator of frequency $\omega_{0}$, so that $\omega_{n m}=$ $\omega_{0}|n-m|$. In this case, taking $l_{n m}=l|n-m|$, with $l$ any positive integer, guarantees that the oscillator reaches the maximal entanglement in a time corresponding to $l$ natural periods. For systems in which condition $(35)$ is not met, the maximal entanglement is reached only in the limit $T \rightarrow \infty$.

Now, the first sum in Eq. (34) runs over all pair of indices $(n, m)$ such that $\omega_{n m} \neq 0$, that is, such that $E_{n} \neq E_{m}$. In the particular case in which there is no degeneracy, this is the same as summing over all pair of indices $(n, m)$ with $n \neq m$, whence we can write

$$
\mathcal{E}_{\max }=\sum_{\substack{n m \\(n \neq m)}}\left|a_{n} a_{m}\right|^{2}=2 \sum_{\substack{n m \\(n<m)}}\left|a_{n} a_{m}\right|^{2} .
$$

If this particular non-degenerate spectrum is also finite, it is intuitively clear that Eq. (36) is maximal for $a_{n}=$ $1 / \sqrt{N}$ for all $n$, where $N$ is the number of terms in the expansion (30) (number of nonzero coefficients). In this (equally weighted) case Eq. (36) leads to

$$
\left.\mathcal{E}_{\max }\right|_{a_{n}^{2}=1 / N}=2 \cdot \frac{1}{N^{2}} \cdot \frac{N(N-1)}{2}=1-\frac{1}{N} .
$$

Therefore, the maximal entanglement increases (tends to 1) as the number of terms in (30) increases, showing that the least information we have on the particular energy eigenstate state in the wave function (30), the more entangled the system is with the clock, and more varied the evolution will be.

Let us now consider the second equality in Eq. (34), and write it in the form

$$
\begin{aligned}
\mathcal{E}_{\max } & =1-\sum_{\substack{n m \\
\left(E_{n}=E_{m}\right)}}\left|a_{n} a_{m}\right|^{2} \\
& =1-\sum_{E}\left(\sum_{\substack{n \\
\left(E_{n}=E\right)}}\left|a_{n}\right|^{2}\right)\left(\sum_{\substack{m \\
\left(E_{m}=E\right)}}\left|a_{m}\right|^{2}\right) \\
& =1-\sum_{E} P^{2}(E),
\end{aligned}
$$

where we introduced the probability distribution

$$
P(E)=\sum_{\substack{m \\\left(E_{m}=E\right)}}\left|a_{m}\right|^{2},
$$

which gives the probability of getting the value $E$ when an energy measurement is performed (taking into account degeneracy). The sum in Eq. (38), performed over all indices $\{n\}$, is therefore equivalent to sum over all energies $E_{n}$ (again, taking into account degeneracy), and Eq. (38) can be expressed as

$$
\mathcal{E}_{\text {max }}=1-\sum_{E} P^{2}(E)=S_{L}(E),
$$

where $S_{L}(E)$ stands for the linear entropy associated to the energy measurement. Finally, this gives

$$
\mathcal{E}(T) \leq S_{L}(E), \quad \lim _{T \rightarrow \infty} \mathcal{E}(T)=S_{L}(E),
$$

which is a sort of 'energy-evolution' uncertainty relation.

For a stationary state (a single term in the expansion (30) ) it follows immediately that $\mathcal{E}$ vanishes, as should be expected. The other (non-trivial) minimum of $\mathcal{E}$ is attained, according to Eq. (33), when each term $\operatorname{sinc}^{2}\left(\omega_{n m} T / 2\right)$ reaches its maximum value. In its turn, this occurs at the local maxima or minima of sinc $\left(\omega_{n m} T / 2\right)$, which correspond to those points that satisfy the condition sinc $\left(\omega_{n m} T / 2\right)=\cos \left(\omega_{n m} T / 2\right)$.

\subsection{Entanglement Measure Based on the von Neumann Entropy}

Due to the importance of the von Neumann entropy, discussed in Section 2, it is instructive to analyze here the entanglement indicator based on $S_{v N}$, and given by (see Eq. 11)

$$
\begin{aligned}
\mathcal{E}_{v N} & \equiv S_{v N}\left[\rho_{R}\right]=-\operatorname{Tr}\left[\rho_{R} \ln \rho_{R}\right] \\
& =S_{v N}\left[\rho_{C}\right]=-\operatorname{Tr}\left[\rho_{C} \ln \rho_{C}\right] .
\end{aligned}
$$

For this purpose we are first going to obtain an expression for $\rho_{C}$. From Eq. (17) we get for the complete $R+C$ density matrix:

$$
|\Psi\rangle\left\langle\Psi\left|=\frac{1}{T} \int \Psi(\boldsymbol{x}, t) \Psi^{*}\left(\boldsymbol{x}^{\prime}, t^{\prime}\right)\right| \boldsymbol{x}\right\rangle\left\langle\boldsymbol{x}^{\prime}|| t\right\rangle\left\langle t^{\prime}\right| d \boldsymbol{x} d \boldsymbol{x}^{\prime} d t d t^{\prime},
$$

and consequently the reduced, marginal density matrix of the clock reads

$$
\begin{aligned}
\rho_{C}= & \operatorname{Tr}_{R}|\Psi\rangle\langle\Psi| \\
= & \int\left\langle\boldsymbol{x}^{\prime \prime} \mid \Psi\right\rangle\left\langle\Psi \mid \boldsymbol{x}^{\prime \prime}\right\rangle d \boldsymbol{x}^{\prime \prime} \\
= & \frac{1}{T} \int \Psi(\boldsymbol{x}, t) \Psi^{*}\left(\boldsymbol{x}^{\prime}, t^{\prime}\right)\left\langle\boldsymbol{x}^{\prime \prime} \mid \boldsymbol{x}\right\rangle\left\langle\boldsymbol{x}^{\prime} \mid \boldsymbol{x}^{\prime \prime}\right\rangle \times \\
& \times|t\rangle\left\langle t^{\prime}\right| d \boldsymbol{x} d \boldsymbol{x}^{\prime} d \boldsymbol{x}^{\prime \prime} d t d t^{\prime} \\
= & \frac{1}{T} \int\left[\int \Psi(\boldsymbol{x}, t) \Psi^{*}\left(\boldsymbol{x}, t^{\prime}\right) d \boldsymbol{x}\right]|t\rangle\left\langle t^{\prime}\right| d t d t^{\prime} \\
= & \frac{1}{T} \int\left\langle\widetilde{\Phi}_{t^{\prime}} \mid \widetilde{\Phi}_{t}\right\rangle|t\rangle\left\langle t^{\prime}\right| d t d t^{\prime},
\end{aligned}
$$

where in the last line we used Eq. 29. Resorting now to Eq. 31) we are led to

$$
\rho_{C}=\sum_{n}\left|a_{n}\right|^{2}\left|\alpha_{n}\right\rangle\left\langle\alpha_{n}\right|
$$


with

$$
\left|\alpha_{n}\right\rangle=\frac{1}{\sqrt{T}} \int e^{-\frac{i}{\hbar} E_{n} t}|t\rangle d t
$$

Notice that the states $\left|\alpha_{n}\right\rangle$ are normalized (in the temporal interval $[0, T]$ ), yet are not necessarily orthogonal to each other. Indeed, one has

$$
\left\langle\alpha_{n} \mid \alpha_{n^{\prime}}\right\rangle=\frac{i \hbar}{T\left(E_{n^{\prime}}-E_{n}\right)}\left[e^{\frac{-i T}{\hbar}\left(E_{n}^{\prime}-E_{n}\right)}-1\right],
$$

so in the limit $T \rightarrow \infty$, the states $\left|\alpha_{n}\right\rangle$ corresponding to different energies $E_{n}$ become orthogonal to each other. It is important to realize that, even though the states $\left\{\left|\alpha_{n}\right\rangle\right\}$ are not orthogonal to each other, they are linearly independent.

Let us denote with $\left\{E^{(j)}\right\}=\left\{E^{(0)}, E^{(1)}, \ldots\right\}$ the succession, in increasing order, of different energy values appearing in the set $\left\{E_{n}\right\}=\left\{E_{0}, E_{1}, \ldots\right\}$ of energy eigenvalues. Notice that, while some of the $E_{n}$ 's may be equal due to degeneracy, all the $E^{(j)}$ 's are different, and satisfy the strict inequalities, $E^{(0)}<E^{(1)}<\ldots$. Moreover, in contrast to what happens with the $E_{n}$ 's, the index $j$ appearing in $E^{(j)}$ does not refer to the eigenvalue of the Hamiltonian's $j$-th eigenstate, but rather labels a particular value among the set of energy eigenvalues. This notation allows us to write the sum (45) over the states $n$, as a sum over the energy values $E^{(J)}$ as follows. First we decompose 45 in the form

$$
\begin{aligned}
\rho_{C} & =\sum_{n}\left|a_{n}\right|^{2}\left|\alpha_{n}\right\rangle\left\langle\alpha_{n}\right| \\
& =\sum_{\substack{m \\
\left(E_{m}=E^{(0)}\right)}}\left|a_{m}\right|^{2}\left|\alpha_{m}\right\rangle\left\langle\left.\alpha_{m}\left|+\sum_{\substack{l \\
\left(E_{l}=E^{(1)}\right)}}\right| a_{l}\right|^{2} \mid \alpha_{l}\right\rangle\left\langle\alpha_{l}\right|+\ldots
\end{aligned}
$$

We then observe that in each sum (characterized by a given value of the energy), all the $\left|\alpha_{n}\right\rangle$ 's coincide. Thus, for example, in the first sum $E_{m}=E^{(0)}$ for all $m$, whence

$$
\begin{aligned}
\left|\alpha_{m}\right\rangle & =\frac{1}{\sqrt{T}} \int e^{-\frac{i}{\hbar} E_{m} t}|t\rangle d t \\
& =\frac{1}{\sqrt{T}} \int e^{-\frac{i}{\hbar} E^{(0)}}|t\rangle d t \equiv\left|\alpha_{E^{(0)}}\right\rangle .
\end{aligned}
$$

This gives

$$
\begin{aligned}
\rho_{C}= & \sum_{\substack{m \\
\left(E_{m}=E^{(0)}\right)}}\left|a_{m}\right|^{2}\left|\alpha_{E^{(0)}}\right\rangle\left\langle\alpha_{E^{(0)}}\right|+ \\
& +\sum_{l}\left|a_{l}\right|^{2}\left|\alpha_{E^{(1)}}\right\rangle\left\langle\alpha_{E^{(1)}}\right|+\ldots \\
= & \sum_{E^{(j)}}^{\left(E_{l}=E^{(1)}\right)} \sum_{\substack{n \\
\left(E_{n}=E^{(j)}\right)}}\left|a_{n}\right|^{2}\left|\alpha_{E^{(j)}}\right\rangle\left\langle\alpha_{E^{(j)}}\right| .
\end{aligned}
$$

Finally, resorting to Eq. (39) we arrive at

$$
\rho_{C}=\sum_{E} P(E)\left|\alpha_{E}\right\rangle\left\langle\alpha_{E}\right|
$$

where the sum runs over all different values of the energy (we dropped out the superindex $(j)$ in $E^{(j)}$ ), and any possible degeneracy has been taken into account. The marginal density matrix of the clock is thus a statistical mixture of the pure states $\left|\alpha_{E}\right\rangle$, with statistical weights $P(E)$.

Now, in general it is not possible to obtain a closed expression for the von Neumann entropy $S_{v N}\left[\rho_{C}\right]$, because it depends on the particular form exhibited by the energy spectrum of the Hamiltonian $H_{R}$. However, we can obtain a useful upper bound for $S_{v N}\left[\rho_{C}\right]$, if we assume (as done here) that the Hilbert space of system $R$ has finite dimension, so the sum in (51) is also finite. In general, when one has a convex linear combination of density matrices of a finite-dimensional quantum system, $\rho=\sum_{i} \lambda_{i} \rho_{i}$, with $0 \leq \lambda_{i} \leq 1$ and $\sum_{i} \lambda_{i}=1$, one has that $S_{v N}[\rho] \leq \sum_{i} \lambda_{i} S_{v N}\left[\rho_{i}\right]-\sum_{i} \lambda_{i} \ln \lambda_{i}[32$. Applying this inequality to (51) one gets,

$$
\mathcal{E}_{v N}=S_{v N}\left[\rho_{C}\right] \leq-\sum_{E} P(E) \ln P(E)=S(E),
$$

where we have used that the entropy of a pure state vanishes (so that $S_{v N}\left[\left|\alpha_{E}\right\rangle\left\langle\alpha_{E}\right|\right]=0$ ), and $S(E)$ denotes the Shannon entropy, $S[E]=-\sum_{E} P(E) \ln P(E)$, of the probability distribution $P(E)$ associated with the possible results of measuring the energy of the system $R$. Equation 52 thus shows that the entanglement $\mathcal{E}_{v N}$ is bounded from above by the Shannon entropy, an upper bound similar to the upper bound (41) associated with the linear entropy.

As already mentioned, in the limit $T \rightarrow \infty$, the states $\left|\alpha_{E}\right\rangle$ become orthogonal to each other. It follows from Eq. (51) that in that limit, the values of $P(E)$ coincide with the eigenvalues of $\rho_{C}$, and the inequality in (52) becomes an equality (see Eq. 12 ). Therefore, we have

$$
\lim _{T \rightarrow \infty} \mathcal{E}_{v N}=S(E)
$$

That is, in the limit $T \rightarrow \infty$, the entanglement between the clock and the system $R$ (as measured by the von Neumann entropy of either $\rho_{R}$ or $\rho_{C}$ ), equals the Shannon entropy associated with the measurement of the energy of $R$.

\subsection{Entanglement and energy fluctuations}

We have seen above that in a stationary state, i.e., when the energy dispersion

$$
\sigma_{E}^{2}=\left\langle\Psi\left|\hat{H}_{R}^{2}\right| \Psi\right\rangle-\left\langle\Psi\left|\hat{H}_{R}\right| \Psi\right\rangle^{2}
$$

vanishes, the entanglement $\mathcal{E}$ vanishes as well. This suggests a relation between $\sigma_{E}^{2}$ and $\mathcal{E}$, that we shall now 
explore. Equation (54) in the general state 30 becomes

$$
\begin{aligned}
\sigma_{E}^{2} & =\sum_{n}\left|a_{n}\right|^{2} E_{n}^{2}-\left(\sum_{n}\left|a_{n}\right|^{2} E_{n}\right)^{2} \\
& =\sum_{n m}\left|a_{n} a_{m}\right|^{2} E_{n}^{2}-\sum_{n m}\left|a_{n} a_{m}\right|^{2} E_{n} E_{m} \\
& =\sum_{n m}\left|a_{n} a_{m}\right|^{2} E_{n}\left(E_{n}-E_{m}\right) .
\end{aligned}
$$

Making $n \leftrightarrow m$ in the last line, and combining both expressions we get

$$
\begin{aligned}
\sigma_{E}^{2} & =\frac{1}{2} \sum_{n m}\left|a_{n} a_{m}\right|^{2}\left(E_{n}-E_{m}\right)^{2} \\
& =\frac{\hbar^{2}}{2} \sum_{\substack{n m \\
\left(\omega_{n m} \neq 0\right)}}\left|a_{n} a_{m}\right|^{2} \omega_{n m}^{2} .
\end{aligned}
$$

Now, coming back to Eq. (33), and resorting to the Taylor series of the sinc function

$$
\operatorname{sinc}(z)=\sum_{l=0}^{\infty} \frac{(-1)^{l} z^{2 l}}{(2 l+1) !}
$$

we find that to lowest orden in $T, \mathcal{E}$ becomes

$$
\mathcal{E}(T)=\frac{1}{3} \sum_{\substack{n m \\\left(\omega_{n m} \neq 0\right)}}\left|a_{n} a_{m}\right|^{2}\left(\omega_{n m} T / 2\right)^{2}=\frac{T^{2}}{6 \hbar^{2}} \sigma_{E}^{2},
$$

so the dispersion in the energy determines the initial rate of grow of $\mathcal{E}$.

\subsection{An example. The qubit case}

We now focus on a qubit (two-level) system, considering the expansion (30) with two terms only,

$$
\Psi(\boldsymbol{x}, t)=a_{1} e^{-i E_{1} t / \hbar} \varphi_{1}(\boldsymbol{x})+a_{2} e^{-i E_{2} t / \hbar} \varphi_{2}(\boldsymbol{x}) .
$$

We can compute for this example the marginal density matrix of $R$,

$$
\rho_{R}=\left(\begin{array}{cc}
\left|a_{1}\right|^{2} & e^{\frac{i\left(E_{2}-E_{1}\right) T}{2 \hbar}} \operatorname{sinc} \frac{\omega_{12} T}{2} a_{1} a_{2}^{*} \\
e^{\frac{i\left(E_{1}-E_{2}\right) T}{2 \hbar}} \operatorname{sinc} \frac{\omega_{12} T}{2} a_{1}^{*} a_{2} & \left|a_{2}\right|^{2}
\end{array}\right)
$$

with eigenvalues

$$
\lambda_{1,2}=\frac{1}{2}\left(1 \pm \sqrt{1-4\left|a_{1} a_{2}\right|^{2}\left(1-\operatorname{sinc}^{2} \frac{\omega_{12} T}{2}\right)} .\right.
$$

In this case, Eq. (33) reduces to

$$
\mathcal{E}(T)=2\left|a_{1} a_{2}\right|^{2}\left(1-\operatorname{sinc}^{2} \frac{\omega_{12} T}{2}\right),
$$

and the energy standard deviation $\sigma_{E}=\sqrt{\sigma_{E}^{2}}$ takes the simple form (see Eq. 56 )

$$
\sigma_{E}=\left|a_{1} a_{2}\right| \hbar \omega_{12}
$$

This gives

$$
\mathcal{E}(T)=2\left|a_{1} a_{2}\right|^{2}\left(1-\operatorname{sinc}^{2} \frac{\sigma_{E} T}{2 \hbar\left|a_{1} a_{2}\right|}\right) .
$$

When $T \sigma_{E} / \hbar=2 \pi\left|a_{1} a_{2}\right| l$, with $l=1,2, \ldots, \mathcal{E}(T)$ reaches its maximum value $\mathcal{E}_{\max }=2\left|a_{1} a_{2}\right|^{2}$ (such condition is equivalent to condition (35). Moreover, if $a_{1}=a_{2}=$ $1 / \sqrt{2}, \mathcal{E}(T)$ becomes

$$
\left.\mathcal{E}(T)\right|_{a_{n}^{2}=1 / 2}=\frac{1}{2}\left(1-\operatorname{sinc}^{2} \frac{\sigma_{E} T}{\hbar}\right),
$$

and the maximum value is $\mathcal{E}_{\max }=1 / 2$. Thus, whenever $\sigma_{E} T=\hbar \pi l$, the maximum value of $\mathcal{E}$ is attained for a balanced coherent superposition of (two) energy eigenstates ( of $\hat{H}_{R}$ ).

It is instructive here to consider the von Neumann entropy $S_{v N}$, or equivalently the entanglement indicator $\mathcal{E}_{v N}$ (see Eq. (42), which in this case can be computed directly as follows. By combining Eqs. 61 and 62 we get,

$$
\lambda_{1}=\frac{1}{2}(1+\sqrt{1-2 \mathcal{E}}), \quad \lambda_{2}=\frac{1}{2}(1-\sqrt{1-2 \mathcal{E}}),
$$

and inserting the eigenvalues $\lambda_{1,2}$ into $\mathcal{E}_{v N}=S_{v N}=$ $-\lambda_{1} \ln \lambda_{1}-\lambda_{2} \ln \lambda_{2}$ (see Eq. (12), we obtain a closed expression for $\mathcal{E}_{v N}$ - which measures the entanglement between the system $R$ (in this case the qubit) and the clock - as a function of the entanglement indicator $\mathcal{E}$ :

$$
\begin{aligned}
& \mathcal{E}_{v N}=-\frac{1}{2}(1+\sqrt{1-2 \mathcal{E}}) \ln \left(\frac{1}{2}(1+\sqrt{1-2 \mathcal{E}})\right)- \\
& -\frac{1}{2}(1-\sqrt{1-2 \mathcal{E}}) \ln \left(\frac{1}{2}(1-\sqrt{1-2 \mathcal{E}})\right) .
\end{aligned}
$$

Figure 1 shows that $S_{v N}$ (and, consequently, also $\mathcal{E}_{v N}$ ) is a monotonic increasing function of the entanglement indicator given by the linear entropy $S_{L}$, or equivalently of the average distinguishability discussed at the end of Section 4. This confirms the tight relation between evolution and entanglement, whether the latter is quantitatively assessed using the linear or the von Neumann entropy.

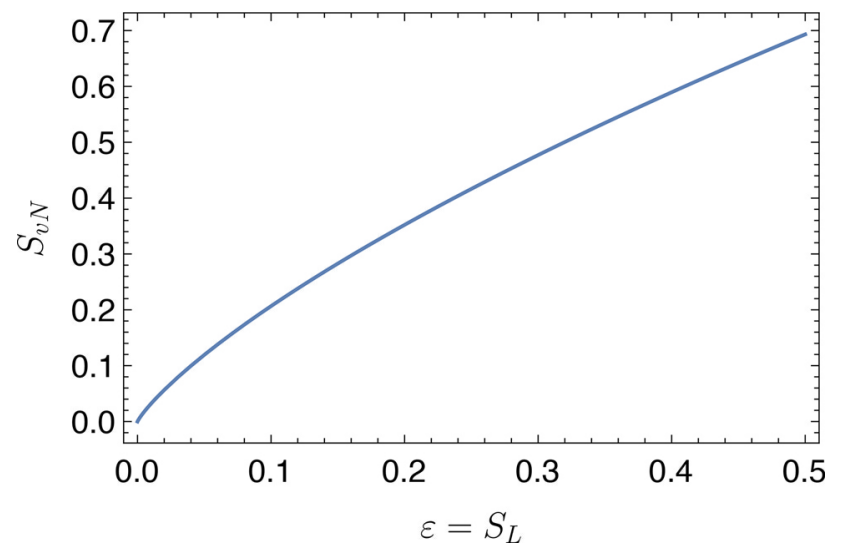

Figure 1: von Neumann entropy as a function of the linear entropy for an evolving qubit system. 


\section{Concluding remarks}

A pedagogical discussion of entanglement within the timeless approach to quantum mechanics was presented. This is a scenario in which evolution and time are not primitive ingredients of the description of the physical world but, rather, emergent phenomena. The Universe is viewed as consisting of a clock $C$ and a system $R$ (the rest of the Universe) that are jointly in a stationary state. The time evolution of the system $R$ arises from the quantum entanglement between the clock $C$ and $R$. Using a minimum of formalism, we showed that there is a direct and quantitative relation between the quantum entanglement exhibited by the $R+C$ composite and the dynamical evolution experienced by the system $R$. Indeed, the entanglement between the clock and the system $R$ closely related to the average distinguishability between the states of $R$ corresponding to different times.

\section{Acknowledgements}

The authors acknowledge the comments from an anonymous reviewer, which helped to improve the manuscript.

A.V.H. acknowledges financial support from DGAPAUNAM through projects PAPIIT IA101918 and IN113720. A.P.M acknowledges the Argentinian agencies SeCyTUNC and CONICET for financial support.

\section{References}

[1] N. Nielsen and I.L. Chuang, Quantum Computation and Quantum Information (Cambridge University Press, Cambridge, 2000).

[2] J.C. Taylor, Hidden Unity in Nature's Laws (Cambridge University Press, Cambridge, 2002).

[3] C.J. Isham, Lectures on Quantum Theory: Mathematical and Structural Foundations (Imperial College Press, London, 1995).

[4] D.V. Schroeder, Am. J. Phys. 85, 812 (2017).

[5] L. Davidovich, Rev. Bras. Ensino Fis. 37, 4205 (2015).

[6] A. Gomez-Rodriguez and J.L. Aragon, Rev. Mex. de Fisica E 58, 61 (2012).

[7] R. Castellanos, R. Franco and J. Silva-Valencia, Rev. Bras. Ensino Fis. 32, 1 (2010).

[8] R.Q. Odendaal and A.R. Plastino, Eur. J. Phys. 31, 193 (2010).

[9] A.C. de la Torre, D. Goyeneche and L. Leitao, Eur. J. Phys. 31, 325 (2010).

[10] S. Curilef, C. Zander and A.R. Plastino, Eur. J. Phys. 27, 1193 (2006).

[11] G.B. Roston, M. Casas, A. Plastino and A.R. Plastino, Eur. J. Phys. 26, 657 (2005).

[12] P. Tommasini, E. Timmermans and A.F.R. Toledo Piza, Am. J. Phys. 66, 881 (1998).

[13] A. Ekert and P.L. Knight, Am. J. Phys. 63, 415 (1995).

[14] D.N. Page and W.K. Wootters, Phys. Rev. D 27, 2885 (1983).

[15] W.K. Wootters, Int. J. Theor. Phys. 23, 701 (1984).

[16] P. Davies, Am. J. Phys. 73, 23 (2005).
[17] B.S. DeWitt, Phys. Rev. 160, 1113 (1967).

[18] D. Atkatz, Am. J. Phys. 62, 619 (1994).

[19] V. Giovannetti, S. Lloyd and K. Maccone, Phys.Rev. D 92, 0450331 (2015).

[20] S. Massar, P. Spindel, A.F. Varon and C. Wunderlich, Phys. Rev. A 92, 030102 (2015).

[21] A. Boette, R. Rossignoli, N. Gigena and M Cerezo, Phys. Rev. A 93, 0621271 (2016).

[22] C. Marletto and V. Vedral, Phys. Rev. D 95, 0435101 (2017).

[23] A. Boette and R. Rossignoli, Phys. Rev. A 98, 0321081 (2018).

[24] P. Rungta, V. Buzek, C.M. Caves, M. Hillery and G.J. Milburn, Phys. Rev. A 64, 042315 (2001).

[25] P. Zanardi, Phys. Rev. A 63, 040304 (2001).

[26] F. Buscemi, P. Bordone and A. Bertoni, Phys. Rev. A 75, 032301 (2007)

[27] M.C. Tichy, P.A. Bouvrie and K. Mølmer, Phys. Rev. Lett. 109, 260403 (2012).

[28] A.P. Majtey, P.A. Bouvrie, A. Valdes-Hernandez and A.R. Plastino, Phys. Rev. A 93, 032335 (2016).

[29] A. Vidiella-Barranco, Phys. Lett. A 260, 335 (1999).

[30] L.C. Malacarne, R.S. Mendes and E.K. Lenzi, Pys. Rev. E 65, 046131 (2002).

[31] N. Huggett, Everywhere and Everywhen: Adventures in Physics and Philosophy (Oxford University Press, Oxford, 2010).

[32] A. Wehrl, Rev. Mod. Phys. 50, 221 (1978). 